\title{
In vitro activity of amphotericin B cochleates against Leishmania chagasi
}

\author{
Aretha Molina Sesana, Renata Monti-Rocha, Solange Alves Vinhas, \\ Carlos Gustavo Morais, Reynaldo Dietze, Elenice Moreira Lemos/ ${ }^{+}$
}

Núcleo de Doenças Infecciosas, Universidade Federal do Espírito Santo, Vitória, ES, Brasil

Cochleate delivery vehicles are a novel lipid-based system with potential for delivery of amphotericin B (AmB). In this study, the efficacy of cochleates was evaluated by examining the in vitro activity of AmB cochleates (CAMB) against Leishmania chagasi in a macrophage model of infection. We demonstrate that CAMB is nontoxic to macrophages at concentrations as high as $2.5 \mu \mathrm{g} / \mathrm{mL}$, whereas the conventional formulation, AmB deoxycholate, showed high toxicity at this concentration. The in vitro activity of CAMB against $\mathrm{L}$. chagasi was found to be similar to that of the reference drug $\mathrm{AmB}$ deoxycholate, with $E D_{50 \mathrm{~s}}$ of $0.017 \mu \mathrm{g} / \mathrm{mL}$ and $0.021 \mu \mathrm{g} / \mathrm{mL}$, respectively. Considering that L. chagasi affects organs amenable to cochleate-mediated delivery of AmB, we hypothesize that CAMB will be an effective lipid system for the treatment of visceral leishmaniasis.

Key words: amphotericin B cochleates - Leishmania chagasi - in vitro activity

Despite its potent activity against leishmaniasis, the toxicity of the conventional formulation of amphotericin $\mathrm{B}(\mathrm{AmB})$ complexed with deoxycholate micelles limits its use in the treatment of visceral leishmaniasis (VL). To improve the therapeutic index of $\mathrm{AmB}$ and reduce its toxicity, new lipid-based formulations have been developed (Robinson \& Nahata 1999). These drug delivery systems, such as liposomal formulations, lipid complexes, lipid emulsions and colloidal dispersions, have been introduced into clinical practice. However, despite their proven success against leishmaniasis (Dietze et al. 1993, Davidson et al. 1996, Sundar et al. 1997) their high cost impedes their frequent use.

Cochleate delivery vehicles are a novel lipid-based system that has potential for AmB delivery. As described by Zarif et al. (2000), cochleates have a unique multilayered structure consisting of a large, continuous, solid lipid bi-layer sheet rolled into a spiral, with no internal aqueous space. Cochleates are stable phospholipid-calcium precipitates comprised mainly of phosphatidylserine, with a mean diameter of $407 \pm 233.8 \mathrm{~nm}$ and molar ratio of 10:1 lipid/AmB when in complex with the drug. Because the entire cochleate structure is a series of solid layers, components within the interior of the cochleate structure are protected from exposure to harsh environmental conditions or enzymes. Given this feature, cochleates should be an ideal system for oral delivery of AmB.

Beside their usefulness as a system to deliver protein, peptide and DNA for vaccine and gene therapy applications (Mannino \& Gould-Fogerite 1997, Zarif \& Mannino 2000), cochleates have also been used as a delivery system for a variety of drugs, including AmB (Santangelo et

+ Corresponding author: lemosem@ndi.ufes.br

Received 30 August 2010

Accepted 12 November 2010 al. 2000, Zarif et al. 2000, Delmas et al. 2002, Syed et al. 2008, Livne et al. 2010). Additionally, a comprehensive patent specifying that Leishmania major-infected mice were orally treated with AmB cochleates (CAMB) was recently published (Mannino 2010). In the present study, the efficacy of cochleates as a delivery vehicle for AmB was evaluated by examining the in vitro activity of CAMB against Leishmania chagasi in a macrophage model of infection. Its activity and toxicity were also compared to a commercially available AmB preparation.

CAMBs were developed as described by Zarif et al. (2000) and provided by BioDelivery Sciences, USA. Amphotericin B deoxycholate (Fungizone ${ }^{\mathrm{TM}}$, Bristol Myers Squibb, France) were purchased commercially in $50 \mathrm{mg}$ vials and were reconstituted according to the manufacturer's instructions. For in vitro experiments, the drugs were prepared by diluting the stock solutions in RPMI 1640 medium containing 10\% of foetal calf serum according to the concentrations required on the day of experiment.

To assess the cytotoxicity of CAMB, peritoneal macrophages were seeded at $8 \times 10^{4}$ cells/well in 96 -well microplates and incubated overnight with a supply of $5 \%$ $\mathrm{CO}_{2}$ at $37^{\circ} \mathrm{C}$. CAMB and $\mathrm{AmB}$ deoxycholate at different concentrations $(5-0.625 \mu \mathrm{g} / \mathrm{mL})$ were dispensed in triplicate, with three wells without drug acting as the control. The plates were incubated for $72 \mathrm{~h}$ and, to assess cell viability, an 3-(4,5-dimethylthiazole-2-yl)-2-5-diphenyl tetrazolium bromide (MTT) assay was performed. Briefly, $100 \mu \mathrm{L}$ MTT $(0.5 \mathrm{mg} / \mathrm{mL})$ was added to each well and incubated for $4 \mathrm{~h}$. The plate was spun down at $1,000 \mathrm{~g}$ for $10 \mathrm{~min}$ at $4^{\circ} \mathrm{C}$ and the supernatant was aspirated. Yellow tetrazolium salt that had reduced to blue formazan within cells was then dissolved in $100 \mu \mathrm{L}$ of dimethyl sulfoxide and the optical density at $570 \mathrm{~nm}$ was determined. The results were expressed as percentage of viable cells compared with untreated control wells.

The in vitro efficacy of CAMB against $L$. chagasi was evaluated by amastigote-macrophage assay. Peritoneal macrophages of Swiss mice were plated in Labtek 
16-well tissue culture slides (Nunc, NY, USA) and were allowed to adhere for $24 \mathrm{~h}$ in an incubator with a supply of $5 \% \mathrm{CO}_{2}$ at $37^{\circ} \mathrm{C}$. Adherent macrophages were infected with late-log phase promastigotes of $L$. chagasi $(\mathrm{MHOM} / \mathrm{BR} / 74 / \mathrm{PP} 75)$ at a ratio of 7:1 promastigotes: macrophage. After $24 \mathrm{~h}$ of incubation, free promastigotes were removed and the culture was exposed to drugs over a dose range of $0.078-0.002 \mu \mathrm{g} / \mathrm{mL}$, in triplicate at each concentration. After $72 \mathrm{~h}$ of additional incubation, the slides were fixed and stained with Panótico solutions (Laborclin, Paraná, Brazil). At least 100 cells were counted to determine the percentage of infected macrophages. The percent killing was calculated by sigmoidal regression analysis (Prisma 5.03) and the mean $\mathrm{ED}_{50 \mathrm{~s}}$ and $\mathrm{ED}_{90 \mathrm{~s}}$ were determined in three independent assays. An unpaired t-test was applied to determine the significance of CAMB $\mathrm{ED}_{50}$ and $\mathrm{ED}_{90}$ over $\mathrm{AmB}$ deoxycholate in the inhibition of infection in macrophages.

In this study, the toxicity of CAMB on mouse peritoneal macrophages was analyzed and compared to that found for $\mathrm{AmB}$ deoxycholate. The percentage of live cells in cultures treated with CAMB at $5 \mu \mathrm{g} / \mathrm{mL}$ was $86 \%$, while treatment with $\mathrm{AmB}$ deoxycholate at the same concentration resulted in $100 \%$ killing. At concentrations of $2.5 \mu \mathrm{g} / \mathrm{mL}$ CAMB had no toxicity for macrophages, while the same concentration of AmB deoxycholate showed high toxicity, with only $36 \%$ of cells surviving. AmB deoxycholate was non-toxic at a concentration of $0.625 \mu \mathrm{g} / \mathrm{mL}$, demonstrating its high toxicity (Figure). Cochleates lacking drug at $5 \mu \mathrm{g} / \mathrm{mL}$ were non-toxic to cells and there was no difference in cell viability between treated and untreated cells.

The in vitro activity of CAMB against intracellular L. chagasi amastigotes was found to be similar to that of the reference drug AmB deoxycholate, with an $\mathrm{ED}_{50}$ of $0.017 \mu \mathrm{g} / \mathrm{mL}$ for CAMB and $0.021 \mu \mathrm{g} / \mathrm{mL}$ for $\mathrm{AmB}$ deoxycholate. However, CAMB showed higher efficacy compared to the control drug when $\mathrm{ED}_{90 \mathrm{~s}}$ was evaluated (Table). Empty cochleates did not show any anti-leishmanial activity.

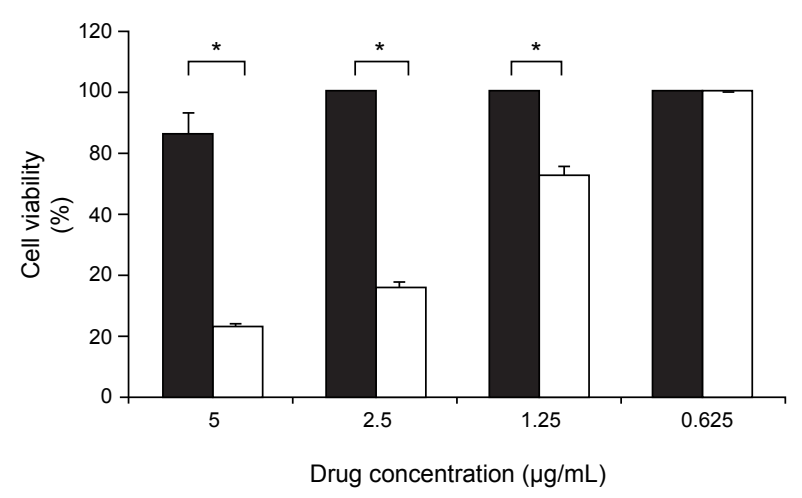

Cytotoxicity assay of amphotericin B (AmB) cochleates ( $\bullet$ ) and AmB deoxycholate ( $\square$ ) using mouse peritoneal macrophages. Values represent the means \pm standard deviation. Asterisks mean significant difference $(\mathrm{p}<0.05)$.
Different studies suggest that drugs incorporated into liposome or lipid formulations are selectively taken up by the phagocytic cells and are concentrated in the liver, spleen and lung (Hiemenz \& Walsh 1996). Moreover, it has been demonstrated that AmB liposomal products have significantly less nephrotoxicity than conventional amphotericin B, even at much higher doses (Walsh et al. 1998).

Encapsulation of AmB into cochleates results in stable, nontoxic and highly efficacious AmB lipid particles, facilitating systemic delivery of AmB. In our study, CAMB treatment was found to be nontoxic to macrophages even at concentrations as high as $2.5 \mu \mathrm{g} /$ $\mathrm{mL}$, whereas $\mathrm{AmB}$ desoxycholate showed high toxicity at this same concentration, confirming the lower toxicity of this system compared to the commercial formulation. In the CAMB formulation, cells do not interact with free $\mathrm{AmB}$, making $\mathrm{CAMB}$ less toxic than desoxycholate AMB. Intact cochleates can induce a perturbation and reordering of the cell membrane, resulting in fusion between the outer layer of the cochleate and the cell membrane. This fusion promotes the delivery of a small amount of the encochleated drug to the cytoplasm of the target cell. Cochleates can also be endocytosed into the cytoplasm of the cell. Once within the low calcium environment of the cytoplasm, the structure of the cochleates alters and the drug is released (Mannino 2010).

In addition to its efficacy in the treatment of experimental candidiasis and aspergillosis (Santangelo et al. 2000, Zarif et al. 2000, Delmas et al. 2002), our results showed that CAMB has a substantial inhibitory action on the growth of $L$. chagasi amastigotes.

Because of the hydrophobicity of AmB molecules, they integrate particularly well with the cochleate struture, providing the additional benefit of protecting $\mathrm{AmB}$ from exposure to harsh environmental conditions or enzymes and facilitating oral administration. Pharmacokinetic studies confirmed that following multiple oral administrations of CAMB, AmB is distributed in target tissues, with the highest concentration of $\mathrm{AmB}$ being in the kidneys, followed by the lungs, liver and spleen (Delmas et al. 2002). The ability of cochleates to deliver systemic $\mathrm{AmB}$ following multiple oral doses highlights the potential of CAMB formulations to treat and prevent invasive infections.

\section{TABLE}

In vitro activities of amphotericin $\mathrm{B}(\mathrm{AmB})$ cochleates (CAMB) and AmB deoxycholate against Leishmania chagasi in mouse peritoneal macrophages

\begin{tabular}{lcc}
\hline Formulation & $\mathrm{ED}_{50}(\mu \mathrm{g} / \mathrm{mL})^{a}$ & $\mathrm{ED}_{90}(\mu \mathrm{g} / \mathrm{mL})^{a}$ \\
\hline CAMB & $0.017 \pm 0.007$ & $0.059 \pm 0.014^{b}$ \\
AmB deoxycholate & $0.021 \pm 0.006$ & $0.090 \pm 0.023^{b}$ \\
\hline
\end{tabular}

$a$ : values are means \pm standard deviation of the means; $b$ : significant difference $(\mathrm{p}<0.05)$. 
In summary, our results demonstrate that CAMB represents a potential lipid-based vehicle for AmB delivery, with high in vitro activity against $L$. chagasi and lower toxicity when compared to the conventional formulation of $\mathrm{AmB}$ deoxycholate. Considering that $L$. chagasi infection affects organs targeted by cochleatemediated delivery of AmB, we can hypothesize that CAMB could be used as a lipid system to treat VL. To investigate this hypothesis, we will further evaluate the potential of CAMB to treat experimental VL using the oral route of administration.

\section{ACKNOWLEDGEMENTS}

To Dr Raphael Mannino, from BioDelivery Science, for providing the amphotericin $\mathrm{B}$ cochleates.

\section{REFERENCES}

Davidson RN, di Martino L, Gradoni L, Giacchino R, Gaeta GB, Pempinello R, Scotti S, Cascio A, Castagnola E, Maisto A, Gramiccia M, di Caprio D, Wilkinson RJ, Bryceson AD 1996. Short-course treatment of visceral leishmaniasis with liposomal amphotericin B (AmBisome). Clin Infect Dis 22: 938-943.

Delmas G, Park S, Chen ZW, Tan F, Kashiwazaki R, Zarif L, Perlin DS 2002. Efficacy of orally delivered cochleates containing amphotericin B in a murine model of aspergillosis. Antimicrob Agents Chemother 46: 2704-2707.

Dietze R, Milan EP, Berman JD, Grogl M, Falqueto A, Feitosa TF, Luz KG, Suassuna FA, Marinho LA, Ksionski G 1993. Treatment of Brazilian kala-azar with a short course of amphocil (amphotericin B cholesterol dispersion). Clin Infect Dis 17: 981-986.

Hiemenz JW, Walsh TJ 1996. Lipid formulations of amphotericin B: recent progress and future directions. Clin Infect Dis 22 (Suppl. 2): S133-S144
Livne L, Epand RF, Papahadjopoulos-Sternberg B, Epand RM, Mor A 2010. OAK-based cochleates as a novel approach to overcome multidrug resistance in bacteria. FASEB J 24: 5092-5101.

Mannino RJ 2010. Cochleate compositions and methods of use. Available from: www.wipo.int/pctdb/en/wo.jsp?WO=2010091090.

Mannino RJ, Gould-Fogerite S 1997. Antigen cochleate formulations for oral and systemic vaccination. In MM Levine (ed.), New generation vaccines, Marcel Dekker, New York, p. 1-9.

Robinson RF, Nahata MC 1999. A comparative review of conventional and lipid formulations of amphotericin B. J Clin Pharm Ther 24: 249-257.

Santangelo R, Paderu P, Delmas G, Chen ZW, Mannino R, Zarif L, Perlin DS 2000. Efficacy of oral cochleate-amphotericin B in a mouse model of systemic candidiasis. Antimicrob Agents Chemother 44: 2356-2360.

Sundar S, Agrawal NK, Sinha PR, Horwith GS, Murray HW 1997. Short-course, low-dose amphotericin B lipid complex therapy for visceral leishmaniasis unresponsive to antimony. Ann Intern Med 127: 133-137.

Syed UM, Woo AF, Plakogiannis F, Jin T, Zhu H 2008. Cochleates bridged by drug molecules. Int J Pharm 363: 118-125.

Walsh TJ, Hiemenz JW, Seibel NL, Perfect JR, Horwith G, Lee L, Silber JL, DiNubile MJ, Reboli A, Bow E, Lister J, Anaissie EJ 1998. Amphotericin B lipid complex for invasive fungal infections: analysis of safety and efficacy in 556 cases. Clin Infect Dis 26: 1383-1396.

Zarif L, Graybill JR, Perlin D, Najvar L, Bocanegra R, Mannino RJ 2000. Antifungal activity of amphotericin B cochleates against Candida albicans infection in a mouse model. Antimicrob Agents Chemother 44: 1463-1469.

Zarif L, Mannino RJ 2000. Cochleates: lipid-based vehicles for gene delivery concept, achievements and future development, In $\mathrm{N}$ Habib (ed.), Cancer gene therapy: past achievements and future challenges, Plenum Publishing Co, London, p. 83-94. 